\title{
Static Magnetic Field Stimulation over Parietal Cortex Enhances Somatosensory Detection in Humans
}

\author{
[Carmen Carrasco-López, ${ }^{1 \star}$ Vanesa Soto-León, ${ }^{1 \star}$ Virginia Céspedes, ${ }^{2}$ Paolo Profice, ${ }^{3}$ Bryan A. Strange $, 4,5$ \\ Guglielmo Foffani, ${ }^{2,6^{*}}$ and ${ }^{\circledR}$ Antonio Oliviero ${ }^{1 *}$ \\ ${ }^{1}$ FENNSI Group and 2Neural Bioengineering Group, Hospital Nacional de Parapléjicos, SESCAM, Toledo 45071, Spain, ${ }^{3}$ Institute of Neurology, Fondazione \\ Policlinico Gemelli, Rome 00168, Italy, ${ }^{4}$ Laboratory for Clinical Neuroscience, Centre of Biomedical Technology, Technical University of Madrid, Madrid \\ 28223, Spain, ${ }^{5}$ Department of Neuroimaging, Reina Sofia Centre for Alzheimer's Research, Madrid 28031, Spain, and ${ }^{6} \mathrm{HM}$ CINAC, Hospital Universitario \\ HM Puerta del Sur, Móstoles, Universidad CEU-San Pablo, Madrid 28938, Spain
}

The role of neuronal oscillations in human somatosensory perception is currently unclear. To address this, here we use noninvasive brain stimulation to artificially modulate cortical network dynamics in the context of neurophysiological and behavioral recordings. We demonstrate that transcranial static magnetic field stimulation (tSMS) over the somatosensory parietal cortex increases oscillatory power specifically in the alpha range, without significantly affecting bottom-up thalamocortical inputs indexed by the early cortical component of somatosensory evoked potentials. Critically, we next show that parietal tSMS enhances the detection of near-threshold somatosensory stimuli. Interestingly, this behavioral improvement reflects a decrease of habituation to somatosensation. Our data therefore provide causal evidence that somatosensory perception depends on parietal alpha activity.

Key words: noninvasive brain stimulation; parietal cortex; static magnet fields; transcranial magnetic stimulation

Significance Statement

Artificially increasing alpha power by placing a powerful magnetic field over the somatosensory cortex overcomes the natural decline in detection probability of a repeated near-threshold sensory stimulus.

\section{Introduction}

Conscious sensory perception is not a passive processing of sensory signals, but depends on the dynamics of cortical networks (Haider and McCormick, 2009). These dynamics are reflected by the oscillatory activity of the brain, which can be studied with neurophysiological techniques such as electroencephalography. Brain oscillations thus offer a window to investigate the links

Received July 2, 2016; revised Feb. 25, 2017; accepted March 1, 2017.

Author contributions: C.C.-L., P.P., B.A.S., G.F., and A.O. designed research; C.C.-L., V.S.-L., and V.C. performed research; C.C.-L., V.S.-L., B.A.S., G.F., and A.O. analyzed data; C.C.-L., B.A.S., G.F., and A.0. wrote the paper.

This work was supported by the "Ministerio de Economía y Competitividad" of Spain and cofinanced by the European Union (FEDER) "A way to make Europe" (projects: SAF2012-39589, SAF2016-80647-R, and Grant SAF2011-27766). We thank J. Gonzalez-Rosa for useful discussion and M. C. Mosqueda-Pozon for technical help.

${ }^{*}$ C.C-L. and V.S.-L. contributed equally to this work.

₹G.F. and A.O. contributed equally to this work.

G.F. and A.O. declare that they are cofounders of the company Neurek SL, which is a spinoff of the Foundation of the Hospital Nacional de Paraplejicos. Moreover, they are inventors listed on the following patents: P201030610 and PCT/ES2011/070290 (patent abandoned). The authors declare no other competing financial interests.

Correspondence should be addressed to either of the following: Guglielmo Foffani, CINAC, Hospital Universitario HM Puerta del Sur, Avenida Carlos V, 70, Mostoles, 28938 Madrid, Spain; E-mail: guglielmo.foffani@gmail.com; or Antonio Oliviero, FENNSI Group, Hospital Nacional de Parapléjicos, Finca La Peraleda s/n, 45071 Toledo, Spain; E-mail:antonio0@sescam.jccm.es.

DOI:10.1523/JNEUROSCI.2123-16.2017

Copyright $\odot 2017$ the authors $\quad 0270-6474 / 17 / 373840-08 \$ 15.00 / 0$ between cortical dynamics and sensory perception in humans (Smith et al., 2006; Supp et al., 2011; Schurger et al., 2015).

Alpha oscillations $(7-13 \mathrm{~Hz})$ represent the most prominent electroencephalographic oscillatory activity, and can be readily recorded over the visual cortex and other cortical areas. Alpha oscillations have an important role on sensory perception. In the visual cortex, for example, it is well established that higher amplitude prestimulus alpha oscillations decrease the perception of subsequent visual stimuli (Hanslmayr et al., 2007; van Dijk et al., 2008; Mathewson et al., 2009). Conversely, in the somatosensory cortex the relationship between alpha oscillations and sensory perception is less clear. While some experiments have reported analogous findings to those obtained in the visual cortex (Weisz et al., 2014; Frey et al., 2016), other studies reported that higher prestimulus alpha oscillations in the parietal cortex increase, instead of decrease, the perception of subsequent somatosensory stimuli (Linkenkaer-Hansen et al., 2004; Ai and Ro, 2014; Hirvonen and Palva, 2016). While these previous studies provide correlative evidence, an alternative approach to determine the causal role of alpha in sensory perception would be to artificially modulate cortical alpha oscillations, via brain stimulation, and measure the effects on sensory perception (Herrmann et al., 2015). 
The use of brain stimulation techniques to study human brain function and to treat neurological and psychiatric disorders is increasing rapidly (Rossini et al., 2015). These techniques typically use either electrical current or changing magnetic fields to stimulate neuronal populations. By contrast, static magnetic fields have only recently been shown to induce effects on the human brain (Oliviero et al., 2011; Roberts et al., 2011), despite early evidence of their interference with neuronal function in animals (Rosen and Lubowsky, 1987; McLean et al., 2003, 2008; Wu and Dickman, 2012; Aguila et al., 2016). Specifically, applying transcranial static magnetic field stimulation (tSMS) over the human precentral cortex reduces the excitability of the motor cortex (Oliviero et al., 2011; Silbert et al., 2013; Nojima et al., 2015; Arias et al., 2017) and can transiently alter the intracortical inhibitory system (Nojima et al., 2015). Moreover, application of tSMS over the visual cortex produces a focal increase in the power of alpha oscillations, inducing the expected decrease in behavioral performance in a highly attention-demanding visual task (Gonzalez-Rosa et al., 2015). The latter results suggest that tSMS could indeed be used to artificially induce focal alpha oscillations at a given cortical area. Thus, in contrast to previous experiments investigating the relationship between alpha oscillations and sensory perception through correlation, tSMS represents a focal neuromodulation approach to determine the causal role of alpha oscillations in sensory perception.

In the present work, we first performed neurophysiological experiments (Experiments 1 and 2) to measure the effects of tSMS over the somatosensory parietal cortex on the ongoing and stimulus-related brain activity by using EEG recordings and electrical somatosensory stimulation. With these experiments, we found that tSMS over the parietal cortex focally increases alpha oscillations, similarly to what we reported for visual cortex (Gonzalez-Rosa et al., 2015), without inducing any significant effects on bottom-up thalamocortical inputs, as measured by the early cortical component of the somatosensory evoked potentials (SEPs), the N20 (Cruccu et al., 2008). We then performed behavioral experiments (Experiment 3 and 4 ) to test the impact of tSMS (used to artificially increase alpha oscillations in parietal cortex) on the detection of near-threshold somatosensory stimuli. Overall, our data suggest that increasing parietal alpha activity enhances somatosensory detection.

\section{Materials and Methods}

A total of 122 right-handed subjects (56 males) with a mean age of 31.6 years (SD, 7.8) participated in our experiments. Volunteers had no history of hormonal, metabolic, cardiovascular, psychiatric, or neurological disorders, and were medication-free at the time of the study. All participants gave their informed consent. The procedures had the approval of the local ethics committee and were conducted in accordance with the Declaration of Helsinki.

\section{Parietal tSMS}

For all experiments, unless noted otherwise, tSMS (real or sham) was delivered over the left parietal cortex. The center of the stimulation was located $3 \mathrm{~cm}$ behind C3 of the 10-20 EEG system (C3'), corresponding approximately to the primary somatosensory cortex. Subjects lay eyes open in a semidarkened room held at a constant temperature. Two metallic cylinders were placed over the left and right parietal cortices to ensure a symmetric setup, as well as a comfortable position for the subjects. The left cylinder could be either real/magnetic or sham/nonmagnetic, whereas the right cylinder was always nonmagnetic (used only for symmetry/balance). One experimenter was dedicated to initial tSMS positioning (and then left the room) and the second experimenter performed data acquisition and subsequent analyses (double-blind design).

The magnet used for tSMS in all experiments was a cylindrical nickel- plated (Ni-Cu-Ni) NdFeB magnet of $60 \mathrm{~mm}$ diameter and $30 \mathrm{~mm}$ thickness, with weight $670 \mathrm{~g}$ (model MAG60r; custom-made by Supermagnete for Neurek SL). The nominal strength of this magnet is $120 \mathrm{~kg}$, with an intensity of the magnetic field at the surface of the magnet, on the cylinder axis, of $0.45 \mathrm{~T}$ (Rivadulla et al., 2014). At $2-3 \mathrm{~cm}$ from the magnet surface (i.e., the approximate depth of the parietal cortex from the scalp), the magnetic field strength of this magnet on the cylinder axis is 120-200 mT (Rivadulla et al., 2014). Nickel-plated nonmagnetic cylinders (MAG60s, Neurek SL) were used for sham stimulation (Rivadulla et al., 2014). We recently verified that tSMS is a safe procedure in humans (Oliviero et al., 2014).

\section{Experiment 1: effects of parietal tSMS on EEG and SEPs}

Subjects and procedure. Seventeen healthy volunteers (11 males; 17 righthanded; mean age, 30.1; SD, 5.9) participated in Experiment 1. Each subject underwent two experimental sessions (double-blind crossover design), one with real stimulation and the other one with sham stimulation, on separate days in randomized order, at the same hour of the day and at least 1 week apart. Subjects were instructed to refrain from moving or talking unless requested to do so. The experimental session included three conditions: (1) baseline (10 $\mathrm{min}),(2)$ intervention (20 $\mathrm{min}),(3)$ postintervention (10 $\mathrm{min}$ ). SEPs were evoked by electrical stimulation of the right median nerve at wrist. Somatosensory stimuli were delivered at $3 \mathrm{~Hz}$ in blocks of $240 \mathrm{~s}$ each. Between each block there was a resting period of $120 \mathrm{~s}$ (Fig. 1A). We refer to these periods without peripheral stimulation as "pauses." One subject was eliminated from the SEP analysis because the P19-N20 complex was not well defined, and four subjects were eliminated from the spectral analysis due to electrical noise artifacts. The final analysis thus included 16 subjects for SEPs and 13 subjects for electroencephalograhic spectra.

Data acquisition. The EEG was continuously recorded from two Ag/ $\mathrm{AgCl}$ electrodes (using a commercial EEG cap) and by digital EEG equipment (SAM 32, MicroMed; high-pass filter, $0.15 \mathrm{~Hz}$; sampling frequency, $2048 \mathrm{~Hz}$ ). The electrodes were placed over $\mathrm{C}^{\prime}$ and $\mathrm{C}^{\prime}{ }^{\prime}(3 \mathrm{~cm}$ posterior from C3 and C4) according to the 10-20 EEG International System. Recordings used a monopolar montage, with the reference electrode placed on the right earlobe and the ground electrode placed on Fpz. Electrode impedance was kept below $5 \mathrm{~K} \Omega$.

Spectral analysis. Data analysis was performed in MATLAB (MathWorks). EEG segments with artifacts (muscular, ocular, or electrical) were excluded by manual inspection. The channel C3'/C4' was analyzed. Power spectral density was estimated using Welch's averaged modified periodogram method (nonoverlapping, Hamming windowing, $0.5 \mathrm{~Hz}$ frequency resolution). The individual alpha frequency (IAF) for each subject was calculated as the frequency within the $8-12 \mathrm{~Hz}$ range of the EEG spectrum showing the maximum power. We calculated the mean spectral power within the range IAF $\pm 2 \mathrm{~Hz}$. We also calculated the mean spectral power in the theta $(3-7 \mathrm{~Hz})$ and in the beta range $(15-29 \mathrm{~Hz})$. The resulting EEG band power was calculated for every 2 min of the 40 min experiment. Spectral estimates of relative band power (alpha, theta, or beta) during intervention and postintervention in channel $i$ were then obtained for each subject by subtracting the log-transformed power at baseline $\left(\mathrm{Pow}_{i, \text { baseline }}\right)$ from the log-transformed band power during intervention $\left(\mathrm{Pow}_{i, \text { intervention }}\right)$, and postintervention $\left(\mathrm{Pow}_{i \text {,post }}\right)$, according to the following formulas: Relative band $\operatorname{power}_{\text {intervention }}(i)=$ $\log \left[\operatorname{Pow}_{\text {intervention }}(i)\right]-\log \left[\operatorname{Pow}_{\text {baseline }}(i)\right]$; Relative band power post $(i)=$ $\log \left[\operatorname{Pow}_{\text {post }}(i)\right]-\log \left[\operatorname{Pow}_{\text {baseline }}(i)\right]$. We separately analyzed the EEG data during peripheral stimulation and in the "pause" periods. We normalized the EEG power during peripheral stimulation to the baseline at the seventh and eighth minutes, and we then analyzed the alpha and beta power every $120 \mathrm{~s}$ at baseline (one time period), during intervention (eight time periods), and after intervention (four time periods). We normalized the EEG power during the "pause" periods to the baseline at the fifth and sixth minutes, and we analyzed the theta, alpha, and beta power every $120 \mathrm{~s}$ during intervention (two time periods) and after intervention (one time period).

SEPs analysis. SEPs were calculated in channel $\mathrm{C}^{\prime} / \mathrm{C}^{\prime}{ }^{\prime}$. The responses delivered after electrical stimulation were averaged every 4 min (blocks of 240 s). The N20 peak was identified on the waveform, and we measured 


\section{Exp. 1}

A

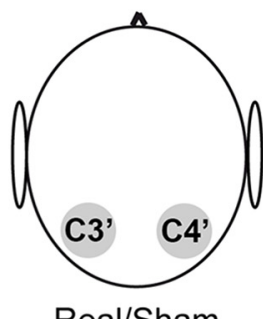

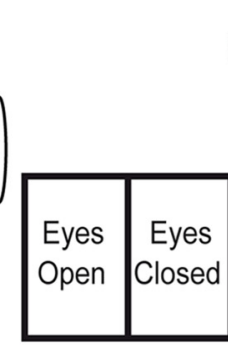

(3')

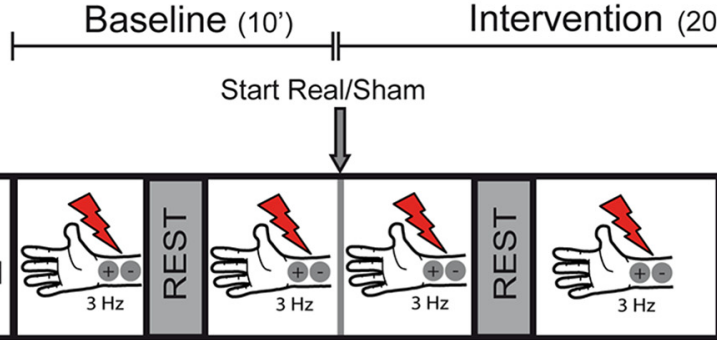

(2')
(2')

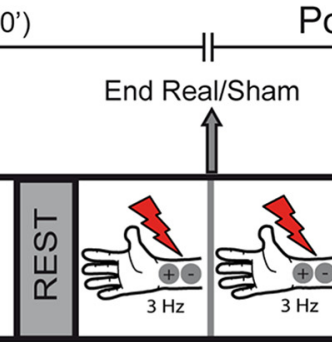

(2')

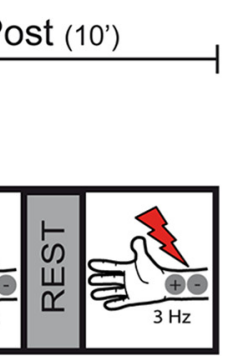

(2')
B

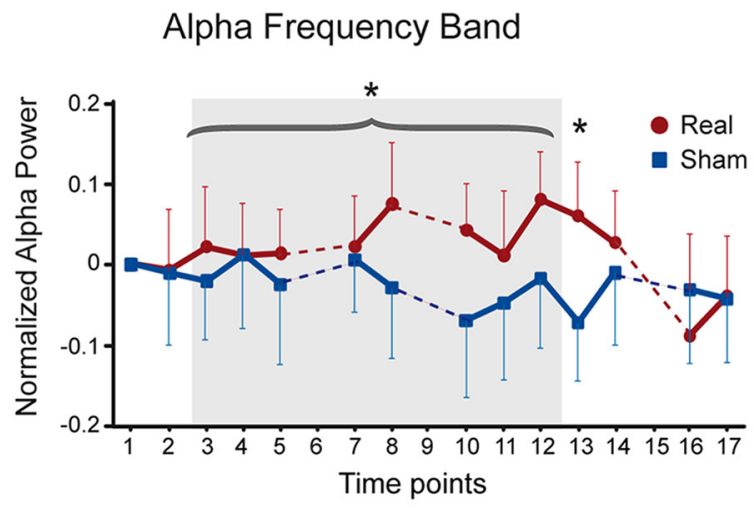

D

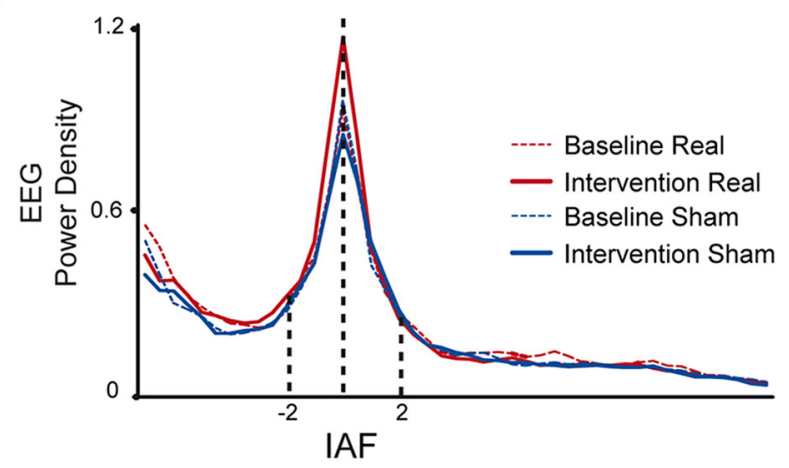

F

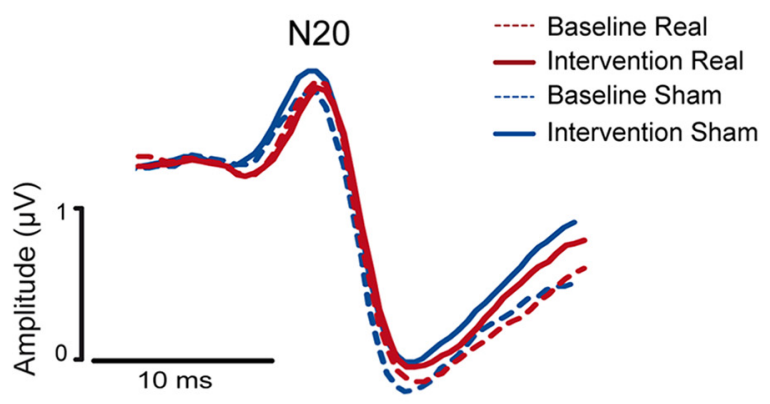

C

Alpha Band at Rest ("Pauses")

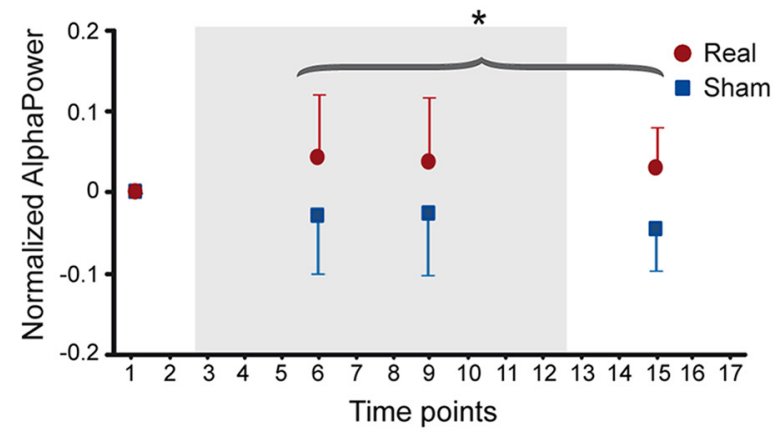

E

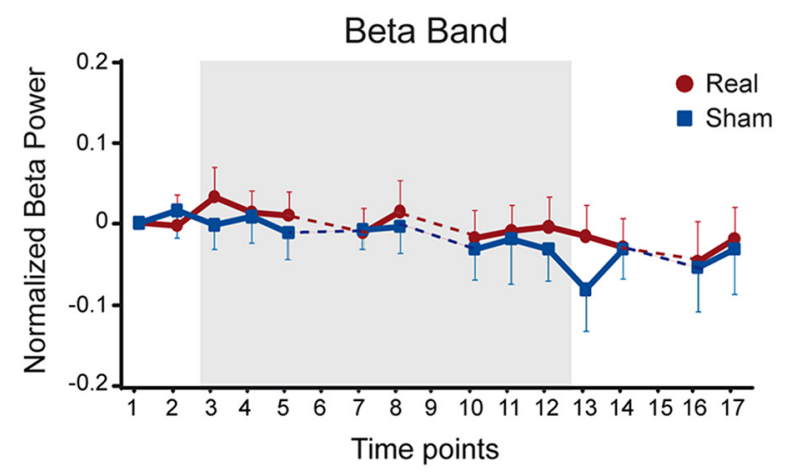

G

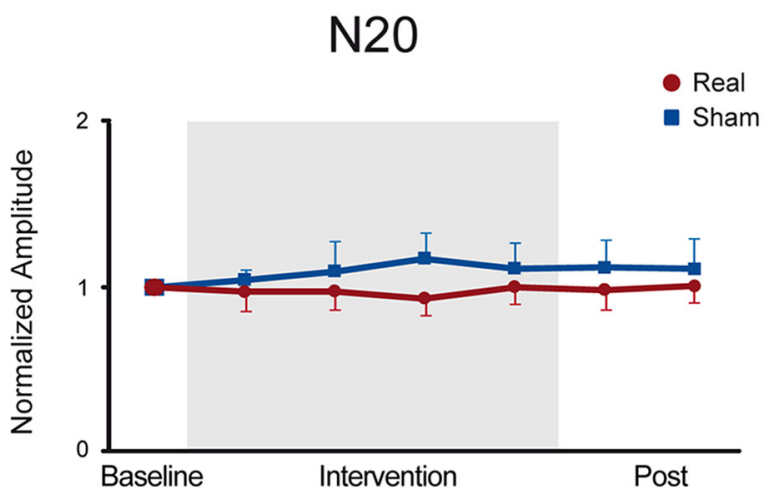

Figure 1. Parietal tSMS increases EEG alpha activity (Experiment 1). A, Schematic illustration of experimental setup. Left, Somatosensory evoked potentials were recorded from C $4^{\prime}$ and C $^{\prime}$ electrodes. Right, The experimental protocol consisted of recording 3 min with eyes open, 3 min with eyes closed, 10 min at baseline, a 20 min intervention, and 10 min after intervention. Left S1 was the area stimulated with real or sham stimulation. Three hertz electrical stimulation at the median right nerve was elicited during baseline, intervention, and postintervention blocks. Rest periods of $2 \mathrm{~min}$ were present at $5-6 \mathrm{~min}$ during baseline, at 7-8 min and 13-14 min during intervention, and at 5- 6 min after intervention. $\boldsymbol{B}$, Time course representation of the log-transformed relative alpha band, normalized to minute $6-7$ of baseline. There was a significant power increment in the alpha band during real stimulation compared to sham stimulation (curly bracket refers to the ANOVA factor Intervention), and the effect persisted $2 \mathrm{~min}$ after the end of the real stimulation (time point 13, the asterisk indicates Tukey's post hoc test (Figure legend continues.) 
its latency and the P19-N20 peak-to-peak amplitude. Data were normalized to the last block of stimulation at baseline.

Statistical analyses. Alpha and beta EEG power during peripheral stimulation were separately entered into two-way repeated-measure ANOVAs with Intervention (real/sham) and Time (13 time points) as main factors. We did not include theta band in this part of the analysis as it was deeply contaminated by the stimulus artifact of the peripheral stimulation. Theta, alpha, and beta EEG power during pause periods were separately entered into two-way repeated-measure ANOVAs with Intervention (real/sham) and Time (three time points) as main factors. SEPs amplitudes were separately entered into two-way repeated-measure ANOVAs with Intervention (real/sham) and Time (six time points) as main factors. In case of significant effects, we used Tukey's test for post hoc analysis or follow-up ANOVAs. All statistical analyses were performed with Statistica software. Results were considered significant at $p<0.05$.

Experiment 2: scalp topography of EEG alpha power in sham and real groups during parietal tSMS at rest

Subjects and procedure. Fifteen healthy volunteers (eight males; 13 righthanded; mean age, 29.4; SD, 6.9) participated in Experiment 2. Each subject underwent two experimental sessions (double-blind crossover design), one with real stimulation and the other with sham stimulation on separate days in randomized order, at the same hour of the day and at least 1 week apart. Subjects were instructed to refrain from moving or talking unless requested to do so. The experimental session consisted of a $10 \mathrm{~min}$ intervention at rest (same tSMS/sham montage as in Experiment 1).

Data acquisition. EEG activity was recorded using a 21 channel EEG cap with built-in $\mathrm{Ag} / \mathrm{AgCl}$ electrodes (EEG Cap Electro-Cap International). The commercial EEG cap was modified to allow the location of the parietal electrodes over $\mathrm{C}^{\prime}$ ' and $\mathrm{C}^{\prime}$ ' ( $3 \mathrm{~cm}$ posterior from $\mathrm{C} 3$ and $\left.\mathrm{C} 4\right)$ according to the 10-20 EEG International System. Electrode positions were located according to the 10/20 EEG International System (Fp1, Fpz, Fp2, F7, F3, Fz, F4, F8, T3, C3, Cz, C4, T4, T5, C3', Pz, C4', T6, O1, Oz, $\mathrm{O}_{2}$ ). Impedance was kept below $5 \mathrm{k} \Omega$. EEG signals were recorded using a monopolar montage with earlobe electrode serving as references and the ground electrode placed on Fpz. The EEG was continuously recorded using a digital EEG equipment (SAM 32, MicroMed; high-pass filter, 0.15 $\mathrm{Hz}$; sampling frequency, $2048 \mathrm{~Hz}$ ).

Data analysis. Data analysis was performed using MATLAB (MathWorks) and EEGLAB (http://sccn.ucsd.edu/eeglab). EEG signals were visually inspected for artifacts, and epochs containing large artifacts such as large blinks or gross motor movements were discarded from further analysis. Power spectral density was estimated using Welch's averaged modified periodogram method (linear detrending, no overlapping, Hamming windowing, $0.5 \mathrm{~Hz}$ frequency resolution). For each condition, single-segment power spectra were computed via fast Fourier transform and then averaged to yield the mean power spectrum.

Statistical analyses. To reduce the number of comparisons, temporal

$\leftarrow$

(Figure legend continued.) after significant Intervention $\times$ Time interaction). Dotted lines represent rest periods. Time points represent 2 min each. $C$, Time course representation of the log-transformed relative alpha band during rest periods ("pauses"), normalized to the minute $4-5$ of baseline. There was a significant power increment in alpha band during real stimulation compared to sham stimulation, and the effect persisted after the end of the real stimulation (curly bracket refers to the ANOVA factor Intervention). Time points represent 2 min each. D, Grand average representation of the EEG power spectral density, for 2 min baseline (dotted lines) and 2 min time point 12 (solid lines), aligned to the IAF of each subject. There was an increase of alpha power after real stimulation but not after sham stimulation. $\boldsymbol{E}$, Time course representation of the log-transformed relative beta band, normalized to minute $6-7$ of baseline. No changes were present between real and sham conditions. Dotted lines represent rest periods. Time points represent 2 min each. $F$, Grand average representation of $\left(3^{\prime} / C 4^{\prime}\right.$ SEPs during baseline in sham condition (dotted blue lines) and real condition (dotted red lines) and during sham intervention (blue lines) and real intervention (red lines). There were no differences in the N2O component of the SEPS. G, Time course representation of the mean of the N2O amplitude. No differences were present in the real and sham group. ${ }^{*} p<0.05$. Error bars indicate $95 \%$ confidence intervals. electrodes were excluded from statistical analyses. Alpha EEG power was entered in a one-way ANOVA with Intervention (real/sham) and Electrode Location (16 channels) as main factors. The real/sham ratio was used for topographic representation of the effects. The topographic distribution was represented with a standard EEG spatial map of 20 electrodes of the Brainstorm software (http://neuroimage.usc.edu/ brainstorm). The electrodes were projected on a $2 \mathrm{D}$ plane with a realistic distribution of the electrodes, and with spatial interpolation of the values between the electrodes.

Experiment 3: effects of parietal tSMS on behavioral performance Subjects and procedure. Fifty right-handed healthy volunteers (29 women; mean age, 32.5; SD, 8.3) participated in Experiment 3. The design was a randomized double-blind, sham-controlled, parallel behavioral study. Three volunteers did not finish the task and were excluded from further analysis. Two subjects were excluded due to excess of false positive behavioral responses, and three subjects were excluded due to detection probability being far from threshold (see Data analyses below). The final analyses were performed on 42 subjects, of which 22 received real tSMS (15 women; mean age, 29.6; SD, 5.8) and 20 received sham tSMS (12 women; mean age, $33.4 ; \mathrm{SD}, 11.4)$. There were no differences between the two groups with respect to gender (two-proportion test, $p=0.58$ ) and age $(t$ test, $p=0.14)$.

The experimental session (30 $\mathrm{min}$ ) consisted of three blocks (10 min each): (1) baseline, (2) intervention (real/sham), and (3) postintervention. Subjects were instructed not to move and to be concentrated in the task throughout the experimental session. The cortical area stimulated with tSMS was the same as in Experiments 1 and $2\left(\mathrm{C}^{\prime}, 3 \mathrm{~cm}\right.$ posterior to the C3 location of the 10-20 EEG International System, approximately corresponding to the left primary somatosensory cortex). The task consisted of saying "yes" into microphone placed next to the mouth as soon as the subject detected an electrical stimulus and to remain silent the rest of the time. In each block, $200 \pm 6$ electrical stimuli were delivered with interstimulus intervals uniformly distributed in the range 1.5-4.5 s, with mean $3 \mathrm{~s}$ (Monto et al., 2008). We placed adhesive electrodes at the distal phalanx of the right index finger to deliver squared-pulse electrical stimuli with a constant intensity current throughout the experimental session. The square electrical stimulus duration was $200 \mu$ s in length, and the intensity of the electrical current was adjusted before starting the experiment for each subject at his own sensory threshold ( $\sim 50 \%$ of trials were detected). This value was fixed for the rest of the experiment.

Data acquisition. Electrical stimuli were triggered by Spike2 7.12 software (bandpass filter, 3-3000 Hz; sampling frequency, $10 \mathrm{kHz}$ ). The same software was used to record the answers of the subjects (using a commercial microphone connected to an input channel of the CED1401 analog-digital converter).

Data analyses. Subjects' responses were computed off-line. Every detected stimulus was classified as a positive response, and the detection probability (number of positive responses/number of delivered stimuli) was calculated. We calculated the reaction time (RT) for each response as the latency from trigger to signal deflection in the microphone channel. Responses within $0.1-1.5 \mathrm{~s}$ after stimulus were considered positive. The absence of a response was classified as negative, and responses outside the $0.1-1.5 \mathrm{~s}$ poststimulus interval were considered as false positives. Subjects with $>3 \%$ of false positives at baseline were excluded (two subjects). We then calculated the detection probability as the number of positive responses/number of delivered stimuli. Subjects with $<0.2$ or $>0.8$ detection probability at baseline were considered not near-threshold and excluded (three subjects).

Statistical analyses. Detection probabilities and reaction times-one value per 10 min block-were separately entered into two-way repeatedmeasure ANOVAs, with Intervention (real/sham) and Time (baseline, during and after the intervention) as main factors. Data were also analyzed in two values per block and subsequently entered into separate two-way repeated-measure ANOVAs in each block (baseline, intervention, and postintervention) with Intervention (real/sham) and Time (two points) as main factors. In case of significant effects, we used Tukey's test for post hoc analysis. All statistical analyses were performed with Statistica software. Results were considered significant at $p<0.05$. 


\section{Experiment 4: specific effects of parietal tSMS on}

behavioral performance

Subjects and procedure. Forty right-handed healthy volunteers (24 women; mean age, 32.8; SD, 8.2) participated in Experiment 4. The design was a randomized double-blind, parallel behavioral study. In this experiment, real tSMS was applied over parietal cortex (in the same way as in the Experiment 3) or over the contalateral prefrontal cortex (over the F4 EEG electrode location). Four subjects were excluded due to excess of false positive responses. Three subjects were excluded due to detection probability being far from threshold. The final analyses were performed on 33 subjects, of which 18 received real tSMS over the parietal cortex (11 women; mean age, $31.3 ; \mathrm{SD}, 9.1$ ) and 15 received real tSMS over the prefrontal cortex (11 women; mean age, 37.7; SD, 5.2).

As in Experiment 3, the experimental session $(30 \mathrm{~min})$ consisted of three blocks (10 min each): (1) baseline, (2) intervention (real parietal tSMS/real prefrontal tSMS), and (3) postintervention. Subjects were instructed not to move and to be concentrated on the task throughout the experimental session. The subjects always had two cylinders located over the target areas, one magnetic for real tSMS and the other nonmagnetic. Real tSMS could be either over the prefrontal cortex or over the parietal cortex, and the subjects and the examiner were blinded about the location of the real tSMS. Subjects were not informed about the lack of sham condition in the experimental design. At the end of the experimental session, subjects were asked whether they thought they received real or sham stimulation (they were not asked about the stimulated location). The task and the finger electrical stimulation were identical to that in Experiment 3. Data acquisition was performed as in Experiment 3.

Statistical analyses. Detection probabilities-one value per $10 \mathrm{~min}$ block-were entered into a two-way repeated-measure ANOVAs, with Intervention Location (real parietal tSMS/real prefrontal tSMS) and Time (baseline, during and after the intervention) as main factors. All statistical analyses were performed with Statistica software. Results were considered significant at $p<0.05$.

\section{Results}

We conducted two main separate experiments (Experiments 1 and 3), each using a sham-controlled, double-blind design. Experiment 1 evaluated the effects of left parietal tSMS on EEG and SEPs (neurophysiological experiment) in one group of subjects ( $n=16$ for SEPs, $n=13$ for EEG) that received both real and sham tSMS in two experimental sessions in randomized order (crossover design). Experiment 3 evaluated the effects of left parietal tSMS on the detection of near-threshold somatosensory stimuli (behavioral experiment) in two groups of subjects that were randomly assigned to receive either real $(n=22)$ or sham tSMS $(n=20)$ in one experimental session (parallel design).

Furthermore, we conducted two control experiments (Experiments 2 and 4). Experiment 2 was conducted to verify the spatial focality of alpha EEG changes induced by parietal tSMS (neurophysiological control) in one group of subjects $(n=15)$ that received both real and sham parietal tSMS in two experimental sessions in randomized order (double-blind crossover design). Experiment 4 evaluated the site specificity of tSMS effects on the detection of near-threshold somatosensory stimuli (behavioral control) in two groups of subjects that were randomly assigned to receive either real left parietal tSMS $(n=18)$ or real right prefrontal tSMS $(n=15)$ in one experimental session (parallel design).

At the end of each experimental session, subjects were interrogated about the type the stimulation (real or sham) they were exposed to. Subjects were not able to identify whether the stimulation was real or sham (Experiment 1, correct guesses, 42.4\%; Pearson's $\chi^{2}=0.8, p=0.37$; Experiment 2, correct guesses, 42.3\%; Pearson's $\chi^{2}=0.6, p=0.43$; Experiment 3, correct guesses, 59.0\%; Pearson's $\chi^{2}=1.7, p=0.19$; Experiment 4 , correct guesses, 39.4\%; Pearson's $\left.\chi^{2}=1.6, p=0.20\right)$. Note that in
Experiment 4 there was no sham condition, but subjects were unaware of this.

None of the subjects reported any adverse event during or after tSMS.

\section{Experiment 1: effects of parietal tSMS on EEG and SEPs}

In the neurophysiological experiment (Experiment 1), we first separately quantified the power in the alpha band during somatosensory stimulation and during intervening rest periods (pauses; Fig. 1A). We observed that the EEG power in the alpha band, calculated during somatosensory stimulation, was increased during and after exposure to tSMS (Fig. $1 B-D$ ). Specifically, while no differences were found in alpha power at baseline between the real and sham tSMS (paired $t$ test, $p=0.95$ ), significant difference between groups (real/sham) was observed during the intervention (Intervention, $F_{(1,12)}=4.8, p=0.0487$ ). The increase of alpha power with real tSMS was still present in the postintervention period, but it gradually returned to the baseline value (Time, $F_{(3,36)}=4.4, p=0.0096$; Intervention $\times$ Time, $F_{(3,36)}=4.2$, $p=0.0125$; Tukey's test, time point 13 , real vs sham, $p=0.0341$; Figure $1 B$ ). We obtained similar results during the pauses at rest (Fig. 1C) — an increase of alpha power during and after real tSMS (Intervention, $\left.F_{(1,12)}=8.6, p=0.0124\right)$.

To exclude the possibility that the increased alpha power could be due to a nonspecific increase in the power of the entire EEG spectrum, we next repeated the same analyses but focusing on the theta $(3-7 \mathrm{~Hz})$ and beta bands $(15-29 \mathrm{~Hz})$. Theta and beta bands were separately analyzed. The power of theta oscillations showed no significant differences between real and sham tSMS (Intervention, $F_{(1,12)}=0.7, p=0.4282$; Intervention $\times$ Time, $\left.F_{(2,24)}=0.22, p=0.8059\right)$. The power of beta oscillations decreased over time (Time, $F_{(12,144)}=4.5, p<0.0001$ ), with no difference between real and sham tSMS (Intervention $\times$ Time, $F_{(12,144)}=1.0, p=0.46$; Fig. $\left.1 E\right)$. This result supports the frequency specificity of the effects induced by parietal tSMS on alpha oscillations.

We further tested whether tSMS affected not only ongoing oscillatory activity, but also the bottom-up thalamocortical inputs evoked by somatosensory stimuli, as assessed by the amplitude of the N20 of SEPs in $\mathrm{C}^{\prime} / \mathrm{C}^{\prime}$ ' channel after peripheral electrical stimulation at the right wrist. We did not find significant differences along time (Time, $F_{(5,75)}=0.4, p=0.86$ ) or between interventions (Intervention, $F_{(1,15)}=3.4, p=0.087$ ) for the amplitude of the N20 component (Fig. $1 F, G$ ).

\section{Experiment 2: scalp topography of EEG alpha power in sham and real groups during parietal tSMS at rest}

The increase in EEG alpha power during tSMS was confirmed in Experiment 2 (Intervention $\times$ Electrodes Location, $F_{(15,210)}=$ 2.7, $p=0.0490)$. The ratio real/sham is plotted in Figure 2, showing an increment of power of alpha oscillations over the left parietal cortex, where the real tSMS was applied.

\section{Experiment 3: effects of parietal tSMS on behavioral performance}

In our first behavioral experiment (Experiment 3), subjects were asked to detect near-threshold somatosensory stimuli in three 10 min blocks: before, during, and after application of real or sham parietal tSMS (Fig. 3A). We quantified both detection probability and reaction time in each block. We found that the detection probability was differentially affected by the real and sham tSMS (Intervention $\times$ Time: $F_{(2,80)}=4.89 ; p=0.0098$; Fig. $3 B$ ). Specifically, in the sham group, the detection probability progressively 


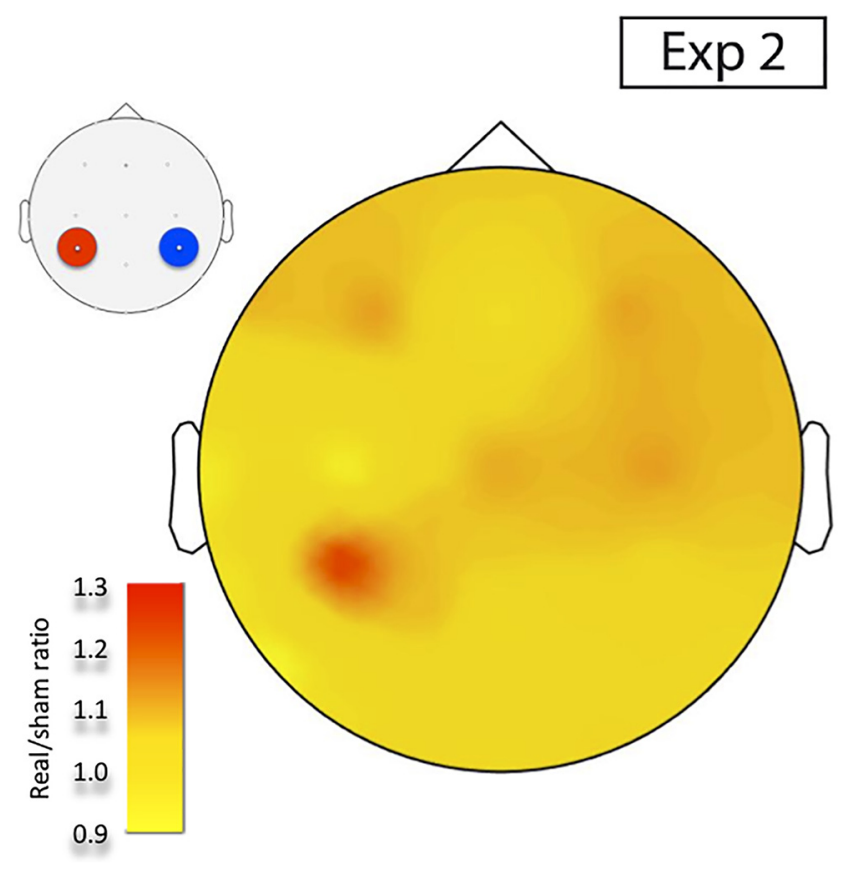

Figure 2. Scalp topography of average EEG alpha power (Experiment 2). Real/sham EEG alpha power ratio of the SSMS intervention session in Experiment 2 (10 min eyes-open resting $\mathrm{EEG}$. Real relative to sham $\mathrm{S}$ SMS over the $\left(3^{\prime}\right.$ electrode produced a focal increase in scalp EEG alpha power of the left parietal cortex.

diminished over time (Tukey's test, baseline vs intervention, $p=0.058$; baseline vs postintervention, $p=0.0001$ ), while no changes were observed in the real group $(p>0.73)$. These data suggest that habituation (Rankin et al., 2009) occurred in the sham group, whereas an improvement of detection, indexed by a decrease of detection probability habituation, occurred in the group undergoing real tSMS.

To further understand this habituation, we quantified the detection probability in subblocks of 5 min (i.e., two values per block analyzed previously). At baseline, habituation was present both in the real and sham groups (Time, $F_{(1,40)}=22.0, p<$ $0.0001)$. However, during the intervention, only the sham group showed a clear habituation (Intervention $\times$ Time, $F_{(1,40)}=5.7$, $p=0.0213$; Tukey's test, Intervention 1, sham vs Intervention 2, sham, $p=0.0002$; Intervention 1 , real vs Intervention 2, real, $p=0.074)$. We thus confirmed that real tSMS improves somatosensory detection so that the physiological habituation is reduced during the intervention. In fact, the detection probability difference between the second and first 5 min subblocks during the intervention was greater in the sham compared with the real group ( $t$ test, $p=0.021$ ). In the postintervention period, habituation was again present in both groups (Time, $F_{(1,40)}=26.3$, $p<0.0001)$.

Finally, we found that the differences in detection probability were paralleled by differences in RT (Intervention $\times$ Time, $F_{(2,80)}=$ $4.69 ; p=0.0118$ ). Specifically, RT became progressively slower in the sham group (follow-up ANOVA, Time, $F_{(2,38)}=3.6, p=0.0363$; Tukey's test, baseline, sham vs postintervention, sham, $p=0.0279$ ), but not in the real group (Time, $F_{(2,42)}=1.32, p=0.28$; Fig. $3 D$ ).

\section{Experiment 4: specific effects of parietal tSMS on behavioral performance}

In the second behavioral experiment (Experiment 4), subjects were asked to detect near-threshold somatosensory stimuli in three 10 min blocks: before, during, and after application of real tSMS applied over the parietal cortex (same location as in Experiment 3) or the prefrontal cortex. We quantified the detection probability in each block. We found that the detection probability was differentially affected by the parietal and prefrontal application of real tSMS (Intervention $\times$ Time, $F_{(2,62)}=3.2$; $p=0.0472$; Fig. 4). These data confirm that habituation (Rankin et al., 2009) occurred with prefrontal tSMS, whereas an improvement of detection associated with reduction of habituation occurred with parietal tSMS. We thus confirmed that real tSMS over the parietal cortex improves somatosensory detection so that the physiological habituation is reduced. The same kind of intervention delivered over the prefrontal cortex is ineffective in modifying sensory stimulus detection.

\section{Discussion}

The main findings of the present work are (1) that parietal tSMS increases alpha EEG oscillations in the parietal cortex, without affecting early evoked potentials and (2) that parietal tSMS improves the detection of near-threshold somatosensory stimuli by interfering with physiological habituation. In a previous paper, we experimentally discarded the possibility that the increased alpha oscillations could be due to artifactual interference between tSMS and the EEG recording system (Gonzalez-Rosa et al., 2015). Thus, the behavioral improvement induced by tSMS may be due to a change of the cortical state, at least partly reflected by the increase in alpha EEG oscillations.

A potential study limitation is that we did not measure alpha oscillations and behavior simultaneously, which might help clarifying whether the relation between increased alpha and improved behavior is monotonic (Linkenkaer-Hansen et al., 2004; Ai and Ro, 2014). However, such experiment would be highly demanding due to high number of stimuli needed, and not necessarily conclusive due to the observed habituation (which was not investigated in previous somatosensory studies using the same behavioral protocol). In any case, our data suggest that globally increasing alpha oscillations with a focal neuromodulation approach improves somatosensory detection.

As an alternative noninvasive brain stimulation strategy, we could have used a technique that directly entrains alpha oscillations, such as $10 \mathrm{~Hz}$ transcranial alternating current stimulation (tACS) or $10 \mathrm{~Hz}$ repetitive transcranial magnetic stimulation (rTMS; Zaehle et al., 2010; Helfrich et al., 2014; Herrmann et al., 2015; Vossen et al., 2015). Entraining techniques increase the power of alpha oscillations and also lock their phase. Conversely, tSMS also increases the power of alpha oscillations in a frequency-specific manner (Gonzalez-Rosa et al., 2015), but leaves the phase free to change with its physiological variability. Even though the role of the phase of alpha oscillations in the detection of somatosensory stimuli requires further investigation (Palva et al., 2005; Hirvonen and Palva, 2016), our results suggest that increasing the power of alpha oscillations is sufficient to improve somatosensory detection.

In the visual cortex, it is well established that higher alpha oscillatory power is associated with reduced cortical excitability (Romei et al., 2008a,b) and impaired behavioral performance on visual tasks (Hanslmayr et al., 2007; van Dijk et al., 2008; Mathewson et al., 2009). Accordingly, we showed previously that tSMS over visual cortex specifically increases occipital alpha oscillations while impairing visual search performance (Gonzalez-Rosa et al., 2015). In the present work, the same technique-tSMS — induces a similar increase of alpha oscillations when applied to the parietal cortex, associated with improved, rather than impaired, somatosensory detection. Importantly, this improved somatosensory detection is 


\section{Exp 3}

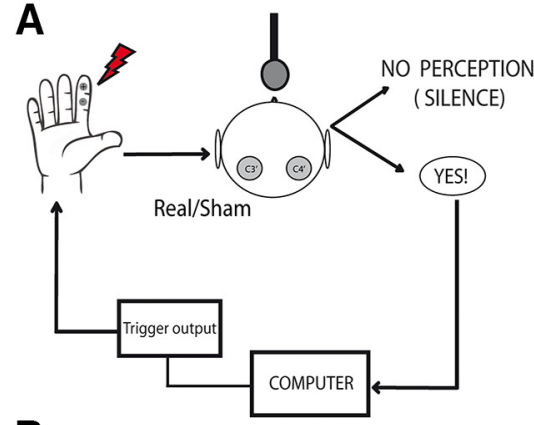

B

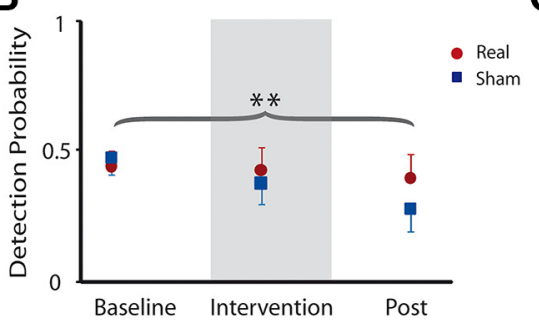

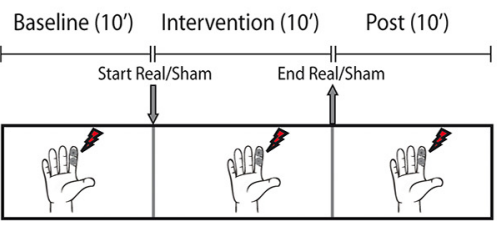

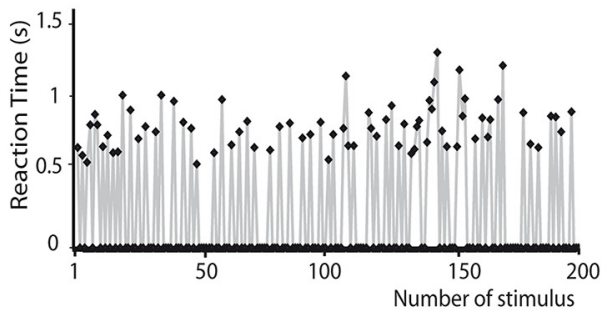

C

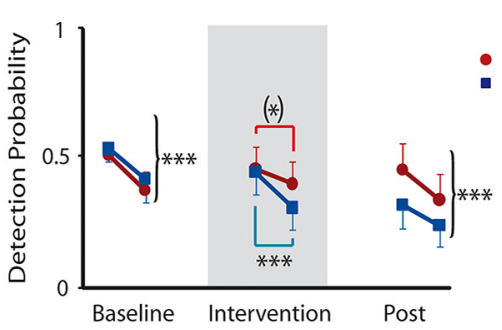

D

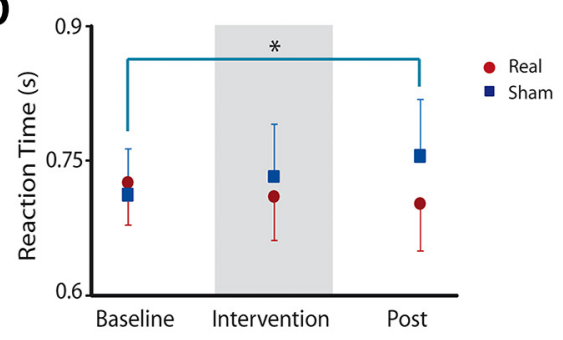

Figure 3. Parietal tSMS increases detection of electrical stimuli (Experiment 3). $\boldsymbol{A}$, Schematic illustration of experimental paradigm. Left, Participants indicated the detection of electrical stimuli at the right index finger by saying "yes" at the microphone, which was recorded by the same computer that sent the triggers. Real or sham stimulation was located over the left $\$ 1$. Center, The experimental protocol was divided into three blocks (baseline, intervention, and postintervention), 10 min each. Right, The sequence of detected stimuli (positive reaction time) and undetected stimuli (zeros) during baseline at near-threshold stimulation in a representative subject. $\boldsymbol{B}$, Time course of stimulus detection probability (1 time point per 10 min block). Detection probability decreased over time in the sham group but not in the real group (curly bracket refers to ANOVA interaction Intervention $\times$ Time). ${ }^{* *} p<0.01$. C, Time course of the stimulus detection probability to study the habituation ( 2 time points per block, 5 min each). At baseline, the detection probability in both groups showed a significant habituation (curly brackets refer to ANOVA factor Time), whereas during the intervention the habituation was strong in the sham group but less clear in the real group (square brackets refer to Tukey's post hoc tests after significant Intervention $\times$ Time interaction). After intervention, habituation was again present in both groups. ${ }^{*} p<0.1 ;{ }^{* * *} p<0.001$. D, Time course of reaction times (1 time point per 10 min block). RTs became progressively slower in the sham group but not in the real group (square bracket refers to Tukey's post hoc tests after significant Intervention $\times$ Time interaction). ${ }^{*} p<0.05$. Error bars indicate $95 \%$ confidence intervals.

\section{Exp 4}

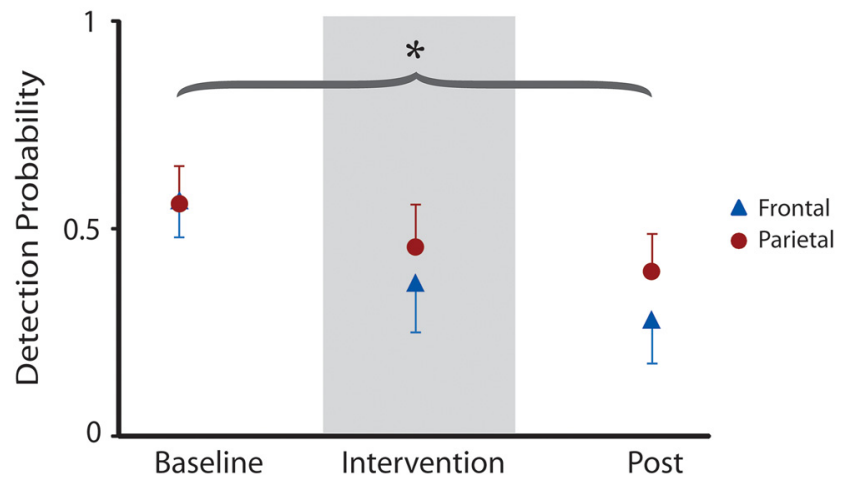

Figure 4. Parietal but not prefrontal tSMS increases detection of electrical stimuli (Experiment 4). The time course of stimulus detection probability (1 time point per $10 \mathrm{~min}$ block) is shown. Detection probability decreased over time in the prefrontal group, but less so in the parietal group (curly bracket refers to ANOVA interaction Intervention $\times$ Time). Error bars indicate $95 \%$ confidence intervals. ${ }^{*} p<0.05$.

unlikely to be due to tSMS-dependent increases of thalamocortical inputs, as the N20 SEP amplitude did not increase with tSMS, consistent with previous results obtained with a somewhat different tSMS protocol (Kirimoto et al., 2014). The N20 is generated when the main inputs of somatosensory volleys reach the primary somatosensory cortex (S1) (Cruccu et al., 2008). It is thus reasonable to speculate that a transient synchronization of cortical neural genera- tors of alpha activity might be mechanistically responsible for the improvement of somatosensory detection induced by tSMS. Additional mechanisms can obviously not be excluded.

Our data suggest that the critical sensory effect of tSMS is the interference with habituation processes. Very little is known about the ability of noninvasive brain stimulation techniques to manipulate sensory habituation (D’Agata et al., 2015). Our results point toward an intriguing connection between sensory habituation and alpha activity. This connection might help explain the highly nonlinear relationship between alpha oscillation and somatosensory behavior reported in some studies (LinkenkaerHansen et al., 2004; Ai and Ro, 2014), as well as the differences between studies using different protocols in the sign of the correlation between parietal alpha and behavioral performance (Weisz et al., 2014; Frey et al., 2016; Hirvonen and Palva, 2016). Further investigations are needed to clarify these issues.

The double-blind sham-controlled design of our experiments rules out the possibility that our results are due to the awareness of tSMS, experimenter bias, arousal decay, or fatigue over the time course of the experiments. Even though the exact mechanisms of these alterations remain to be fully established, the present data show that parietal tSMS affects both alpha EEG activity and somatosensory behavioral performance. Compared with other noninvasive neuromodulation techniques, such as transcranial direct current stimulation, tACS, or rTMS (Paulus et al., 2013), tSMS induces these electrophysiological and behavioral effects without using any electrical current or time-varying magnetic pulses. We conclude that focal static magnetic fields can interfere with normal brain function even at an intensity of $120-$ 
$200 \mathrm{mT}$ (at $2-3 \mathrm{~cm}$ from the magnet surface), offering a novel technique that is both portable and inexpensive to influence human brain activity and behavior.

\section{References}

Aguila J, Cudeiro J, Rivadulla C (2016) Effects of Static Magnetic Fields on the Visual Cortex: reversible Visual Deficits and Reduction of Neuronal Activity. Cereb Cortex 26:628-638. Medline

Ai L, Ro T (2014) The phase of prestimulus alpha oscillations affects tactile perception. J Neurophysiol 111:1300-1307. CrossRef Medline

Arias P, Adán-Arcay L, Puerta-Catoira B, Madrid A, Cudeiro J (2017) Transcranial static magnetic field stimulation of M1 reduces corticospinal excitability without distorting sensorimotor integration in humans. Brain Stimul 10:340-342. CrossRef

Cruccu G, Aminoff MJ, Curio G, Guerit JM, Kakigi R, Mauguiere F, Rossini PM, Treede RD, Garcia-Larrea L (2008) Recommendations for the clinical use of somatosensory-evoked potentials. Clin Neurophysiol 119: 1705-1719. CrossRef Medline

D’Agata F, Cicerale A, Mingolla A, Caroppo P, Orsi L, Mortara P, Troni W, Pinessi L (2015) Double-cone coil TMS stimulation of the medial cortex inhibits central pain habituation. PLoS One 10:e0128765. CrossRef Medline

Frey JN, Ruhnau P, Leske S, Siegel M, Braun C, Weisz N (2016) The tactile window to consciousness is characterized by frequency-specific integration and segregation of the primary somatosensory cortex. Sci Rep 6:20805. CrossRef Medline

Gonzalez-Rosa JJ, Soto-Leon V, Real P, Carrasco-Lopez C, Foffani G, Strange BA, Oliviero A (2015) Static magnetic field stimulation over the visual cortex increases alpha oscillations and slows visual search in humans. J Neurosci 35:9182-9193. CrossRef Medline

Haider B, McCormick DA (2009) Rapid neocortical dynamics: cellular and network mechanisms. Neuron 62:171-189. CrossRef Medline

Hanslmayr S, Aslan A, Staudigl T, Klimesch W, Herrmann CS, Bäuml KH (2007) Prestimulus oscillations predict visual perception performance between and within subjects. Neuroimage 37:1465-1473. CrossRef Medline

Helfrich RF, Schneider TR, Rach S, Trautmann-Lengsfeld SA, Engel AK, Herrmann CS (2014) Entrainment of brain oscillations by transcranial alternating current stimulation. Curr Biol 24:333-339. CrossRef Medline

Herrmann CS, Strüber D, Helfrich RF, Engel AK (2015) EEG oscillations: from correlation to causality. Int J Psychophysiol 103:12-21. CrossRef

Hirvonen J, Palva S (2016) Cortical localization of phase and amplitude dynamics predicting access to somatosensory awareness. Hum Brain Mapp 37:311-326. CrossRef Medline

Kirimoto H, Tamaki H, Matsumoto T, Sugawara K, Suzuki M, Oyama M, Onishi H (2014) Effect of transcranial static magnetic field stimulation over the sensorimotor cortex on somatosensory evoked potentials in humans. Brain Stimul 7:836-840. CrossRef Medline

Linkenkaer-Hansen K, Nikulin VV, Palva S, Ilmoniemi RJ, Palva JM (2004) Prestimulus oscillations enhance psychophysical performance in humans. J Neurosci 24:10186-10190. CrossRef Medline

Mathewson KE, Gratton G, Fabiani M, Beck DM, Ro T (2009) To see or not to see: prestimulus alpha phase predicts visual awareness. J Neurosci 29: 2725-2732. CrossRef Medline

McLean MJ, Engström S, Holcomb RR, Sanchez D (2003) A static magnetic field modulates severity of audiogenic seizures and anticonvulsant effects of phenytoin in DBA/2 mice. Epilepsy Res 55:105-116. CrossRef Medline

McLean MJ, Engström S, Qinkun Z, Spankovich C, Polley DB, Polley D (2008) Effects of a static magnetic field on audiogenic seizures in black Swiss mice. Epilepsy Res 80:119-131. CrossRef Medline

Monto S, Palva S, Voipio J, Palva JM (2008) Very slow EEG fluctuations predict the dynamics of stimulus detection and oscillation amplitudes in humans. J Neurosci 28:8268-8272. CrossRef Medline

Nojima I, Koganemaru S, Fukuyama H, Mima T (2015) Static magnetic field can transiently alter the human intracortical inhibitory system. Clin Neurophysiol 126:2314-2319. CrossRef Medline

Oliviero A, Mordillo-Mateos L, Arias P, Panyavin I, Foffani G, Aguilar J (2011) Transcranial static magnetic field stimulation of the human motor cortex. J Physiol 589:4949-4958. CrossRef Medline
Oliviero A, Carrasco-López MC, Campolo M, Perez-Borrego YA, Soto-León V, Gonzalez-Rosa JJ, Higuero AM, Strange BA, Abad-Rodriguez J, Foffani G (2014) Safety study of transcranial static magnetic field stimulation (tSMS) of the human cortex. Brain Stimul 8:481-485. CrossRef

Palva S, Linkenkaer-Hansen K, Näätänen R, Palva JM (2005) Early neural correlates of conscious somatosensory perception. J Neurosci 25:5248 5258. CrossRef Medline

Paulus W, Peterchev AV, Ridding M (2013) Transcranial electric and magnetic stimulation: technique and paradigms. Handb Clin Neurol 116: 329-342. CrossRef Medline

Rankin CH, Abrams T, Barry RJ, Bhatnagar S, Clayton DF, Colombo J, Coppola G, Geyer MA, Glanzman DL, Marsland S, McSweeney FK, Wilson DA, Wu CF, Thompson RF (2009) Habituation revisited: an updated and revised description of the behavioral characteristics of habituation. Neurobiol Learn Mem 92:135-138. CrossRef Medline

Rivadulla C, Foffani G, Oliviero A (2014) Magnetic field strength and reproducibility of neodymium magnets useful for transcranial static magnetic field stimulation of the human cortex. Neuromodulation 17:438-441; discussion 441-442. CrossRef Medline

Roberts DC, Marcelli V, Gillen JS, Carey JP, Della Santina CC, Zee DS (2011) MRI magnetic field stimulates rotational sensors of the brain. Curr Biol 21:1635-1640. CrossRef Medline

Romei V, Rihs T, Brodbeck V, Thut G (2008a) Resting electroencephalogram alpha-power over posterior sites indexes baseline visual cortex excitability. Neuroreport 19:203-208. CrossRef Medline

Romei V, Brodbeck V, Michel C, Amedi A, Pascual-Leone A, Thut G (2008b) Spontaneous fluctuations in posterior alpha-band EEG activity reflect variability in excitability of human visual areas. Cereb Cortex 18:20102018. CrossRef Medline

Rosen AD, Lubowsky J (1987) Magnetic field influence on central nervous system function. Exp Neurol 95:679-687. CrossRef Medline

Rossini PM, Burke D, Chen R, Cohen LG, Daskalakis Z, Di Iorio R, Di Lazzaro V, Ferreri F, Fitzgerald PB, George MS, Hallett M, Lefaucheur JP, Langguth B, Matsumoto H, Miniussi C, Nitsche MA, Pascual-Leone A, Paulus W, Rossi S, Rothwell JC, et al. (2015) Non-invasive electrical and magnetic stimulation of the brain, spinal cord, roots and peripheral nerves: basic principles and procedures for routine clinical and research application. An updated report from an I.F.C.N. Committee. Clin Neurophysiol 126:1071-1107. CrossRef Medline

Schurger A, Sarigiannidis I, Naccache L, Sitt JD, Dehaene S (2015) Cortical activity is more stable when sensory stimuli are consciously perceived. Proc Natl Acad Sci U S A 112:E2083-E2092. CrossRef Medline

Silbert BI, Pevcic DD, Patterson HI, Windnagel KA, Thickbroom GW (2013) Inverse correlation between resting motor threshold and corticomotor excitability after static magnetic stimulation of human motor cortex. Brain Stimul 6:817-820. CrossRef Medline

Smith ML, Gosselin F, Schyns PG (2006) Perceptual moments of conscious visual experience inferred from oscillatory brain activity. Proc Natl Acad Sci U S A 103:5626-5631. CrossRef Medline

Supp GG, Siegel M, Hipp JF, Engel AK (2011) Cortical hypersynchrony predicts breakdown of sensory processing during loss of consciousness. Curr Biol 21:1988-1993. CrossRef Medline

van Dijk H, Schoffelen JM, Oostenveld R, Jensen O (2008) Prestimulus oscillatory activity in the alpha band predicts visual discrimination ability. J Neurosci 28:1816-1823. CrossRef Medline

Vossen A, Gross J, Thut G (2015) Alpha power increase after transcranial alternating current stimulation at alpha frequency ( $\alpha$-tACS) reflects plastic changes rather than entrainment. Brain Stimul 8:499-508. CrossRef Medline

Weisz N, Wühle A, Monittola G, Demarchi G, Frey J, Popov T, Braun C (2014) Prestimulus oscillatory power and connectivity patterns predispose conscious somatosensory perception. Proc Natl Acad Sci U S A 111: E417-E425. CrossRef Medline

Wu LQ, Dickman JD (2012) Neural correlates of a magnetic sense. Science 336:1054-1057. CrossRef Medline

Zaehle T, Rach S, Herrmann CS (2010) Transcranial alternating current stimulation enhances individual alpha activity in human EEG. PLoS One 5:e13766. CrossRef Medline 